\title{
Essential Components of Antimicrobial Gastrointestinal Epithelial Barrier: Specific Interaction of Mucin with an Integral Apical Membrane Protein of Gastric Mucosa
}

\author{
Amalia Slomiany, Maria Grabska and Bronislaw L. Slomiany \\ Research Center, University of Medicine and Dentistry of New Jersey, New Jersey Dental School, \\ Newark, New Jersey, U.S.A. \\ Accepted September 25, 2000.
}

\begin{abstract}
Background: The gastric mucosal protective barrier consists of two essential elements: mucus glycoprotein, mucin, secreted by gastric mucosal cells, and the mucin binding protein (MBP), an integral component of the apical epithelial membrane. The studies described here provide evidence on the structure of MBP, its interaction with mucin, and the susceptibility to phospholipase C (PLC) and Helicobacter pylori protease.

Material and Methods: The rat gastric mucosa was used to isolate mucin and the apical epithelial membranes. A buffered saline extract of the mucosal cells was used for the isolation of mucin and the $1 \%$ Triton X-100-insoluble gastric apical membranes for the preparation of MBP.

Results: The studies on MBP, the mucosal mucin receptor revealed that the protein is anchored in apical membrane through glycosylphosphatidylinositol (GPI). The deamination of MBP with nitrous acid afforded phosphatidyli-
\end{abstract}

nositols (PIs) and a water soluble, $97 \mathrm{kDa}$ glycosylated protein. The in situ studies with untreated rat gastric mucosa and the mucosa depleted of mucin showed that MBP without mucin was susceptible to the proteolytic degradation with pepsin and $H$. pylori proteases, but was not released from the apical membrane by the treatment with bacterial PLC.

Conclusion: The study of carbohydrate ligands for MBP revealed binding of octa- and decasaccharides of gastric mucin. The severe impairment in mucin adhesion to MBP, induced by the diet containing ethanol, supports the conclusion that specific carbohydrate determinants participate in mucin attachment to MBP and epigenetic control of the processes that coordinates its interaction with apical mucosal epithelium in the formation of innate protective barrier.

\section{Introduction}

For decades, an adhesion of the mucus glycoprotein, mucin, to gastrointestinal lumenal surfaces was viewed as a physical property of the large viscous molecules discharged by the mucosal surfaces (1-3). Hence, the investigations focused on mucin as the product of epithelial cells responsible for lubrication of the lumenal gastrointestinal surfaces, protection from hydrogen ion diffusion, and elimination of the discards and byproducts (4). Over the years, this trend overpowered other conclusions on mucus functions (5) and the research on gastric pathologies concentrated on the acid-suppression therapy (6) or mucosal immunodefenses reflected in the production of immunoglobulin A (IgA) (7-9). Even now, when Helicobacter pylori is recognized as the major contributor to gastric ulcers, gastritis, and cancer, the role of mucin in host defense mechanism is still con-

Address correspondence and reprint requests to: Amalia Slomiany, University of Medicine and Dentistry of New Jersey, Research Center C-873, 110 Bergen Street, Newark, NJ 07103-2400, U.S.A. Phone: 973-972-7023; Fax: 973-972-7020 E-mail: slomiaam@umdnj.edu ceived as the nonspecific physicochemical barrier that can trap microbes $(7,10)$. Nonetheless, our studies show that the tight adherence of mucin to the apical surfaces of epithelia is owed to the existence of the specific complex between mucin oligosaccharides and the mucin binding protein (MBP) of the apical mucosal membrane $(11,12)$. The complex of MBP with mucin is weakened by drug-induced changes in mucin oligosaccharide synthesis, and in the absence of mucin, is easily replaced with $H$. pylori lipopolysaccharide (LPS) (12-16). From the studies on the complex sensitivity to the removal of the carbohydrate residues (11), together with the evidence on LPS binding (12) and LPS mimicry of the host antigenic determinants $(17,18)$, we concluded that mucin retention on the epithelial surfaces was specific and attributed to the mucin carbohydrateintegral mucosal membrane protein complex. The fact that the membrane MBP complex with mucin was destroyed by partial deglycosylation, aberrant glycosylation of mucin $(13,15)$, or substituted with bacterial LPS suggested that posttranslationally acquired oligosaccharidic determinants of mucin were seminal to the ability of mucin to interact with 
epithelium and form its innate protective barrier. In the study presented here, we characterize the gastric apical membrane-expressed MBP, isolate the functional complex of MBP with mucin that renders epithelium resistant to $H$. pylori proteases, and show that the complex formation is dependent on interaction of the specific mucin oligosaccharides with the membrane anchored MBP.

\section{Materials and Methods \\ Isolation of Gastric Mucin and Apical Epithelial Membranes}

Briefly, rat gastric mucosa was disrupted gently with the aid of a loose-fitting pestle of glass homogenizer and passed through a screen to remove occasional fragments of muscular submucosa. To remove extracellular mucin from the cell preparation, the disrupted tissue was suspended in buffered saline, centrifuged at $3000 \mathrm{rpm}$ for $2 \mathrm{~min}$, and then washed once again. Mucin was isolated from the combined supernatants by exclusion column chromatography as described in (19). The recovered cells were suspended in five volumes of ice-cold lysis buffer consisting of $0.2 \mathrm{M}$ piperazine- $\mathrm{N}, \mathrm{N}^{\prime}$-bis[2-ethanesulfonic acid] (PIPES), $\mathrm{pH} 6.9,0.15 \mathrm{M} \mathrm{NaCl}, 2 \mathrm{M}$ glycerol, 1\% Triton X-100, $1 \mathrm{mM}$ ethyleneglycol bis ( $\beta$-amimoethylether) $\mathbf{N}, \mathbf{N}, \mathbf{N}^{\prime} \mathbf{N}^{\prime}$-tetraacetate (EGTA) (EGTA), $1 \mathrm{mM} \mathrm{Mg}$ acetate, and the protease inhibitors cocktail containing aprotinin, leupeptin, pepstatin, sodium orthovanadate, and phenylmethylsulfonyl fluoride (PMSF) (20). After $5 \mathrm{~min}$ at $4^{\circ} \mathrm{C}$, the membranes insoluble in the buffered detergent were separated from the soluble material by low speed centrifugation at $3000 \mathrm{rpm}$ for $2 \mathrm{~min}$. The membranes were treated once again with the same buffered detergent and then suspended in PIPESbuffered saline, $\mathrm{pH} 6.5$, containing $1 \%$ Triton $\mathrm{X}-100$, the protease inhibitors specified above, and $40 \%$ sucrose, overlaid with linear gradient of 5-30\% sucrose in PIPES-buffered saline with protease inhibitors cocktail and centrifuged at 39,000 rpm for $18 \mathrm{hr}$ at $4^{\circ} \mathrm{C}$ in a Beckman SW40 Ti rotor (Palo Alto, CA). The glycosphingolipid-, sphingolipid- and glycosylphosphatidylinositol (GPI)-anchored proteinenriched membranes were isolated from the opaque band floating at $15 \%$ sucrose $(20,21)$. The fraction was rinsed with sucrose-free buffer and subjected to solubilization with Triton X-114 (22).

\section{Precondensation of Triton $X-114$}

20 g Triton X-114 containing $16 \mathrm{mg}$ butylated hydroxytoluene was added to $980 \mathrm{ml}$ of $10 \mathrm{mM}$ Tris$\mathrm{HCl}, \mathrm{pH} 7.4$, and $150 \mathrm{mM} \mathrm{NaCl}$ (22). After dissolution at $0^{\circ} \mathrm{C}$, the mixture was incubated overnight at $30^{\circ} \mathrm{C}$. The aqueous phase of the biphasic system was discarded, the detergent phase was mixed twice again with a new portion of buffered saline, and each time the separated aqueous phase discarded. After the third condensation, the detergent concentration of $11.2 \%$ (weight per volume; w/v) was obtained and used as the stock solution of the detergent for all the experiments performed. The presence of the detergent, in the lower phase after three extractions, was measured at $275 \mathrm{~nm}$. During extraction of the samples containing proteins, duplicate samples containing detergent solution only were prepared on ice, incubated at the temperature indicated, and centrifuged at $300 \times \mathrm{g}$. The phase separation was checked visually and an aliquot was removed for the spectrophotometric estimation of the concentration of the Triton X-114 remaining in the upper phase.

Extraction of the Mucosal Membranes with Triton $X-114$ and Identification of MBP

The Triton X-100-insoluble membranes (0.2-1.0 $\mathrm{mg} / \mathrm{ml}$ ) were suspended in $200 \mu \mathrm{l}$ of $10 \mathrm{mM}$ Tris$\mathrm{HCl}, \mathrm{pH} 7.4,150 \mathrm{mM} \mathrm{NaCl}$, and $0.5-1.0 \%$ Triton $\mathrm{X}-114$ at $0^{\circ} \mathrm{C}$. For separation of the phases, a cushion of $6 \%(\mathrm{w} / \mathrm{v})$ sucrose, $10 \mathrm{mM}$ Tris- $\mathrm{HCl}, \mathrm{pH} 7.4,150 \mathrm{mM}$ $\mathrm{NaCl}$, and $0.06 \%$ Triton X-114 (300 $\mu \mathrm{l})$ was placed at the bottom of a $1.5 \mathrm{ml}$ conical Eppendorf microfuge tube (Sigma Chemical Company, St. Louis, MO). The clear sample was then overlaid on the sucrose cushion and incubated $3-5 \mathrm{~min}$ at $30^{\circ} \mathrm{C}$. After clouding of the solution occurred, the tubes were centrifuged at room temperature for 3-5 $\mathrm{min}$ at $300 \times \mathrm{g}$. The upper aqueous phase was remo-ved, mixed with a fresh portion of $0.5 \%$ Triton $\mathrm{X}-114$ solution, cooled to $0^{\circ} \mathrm{C}$, and overlaid again on the sucrose cushion used in the first separation. After repeating the incubation at $30^{\circ} \mathrm{C}$ and centrifugation, the detergent droplet from the bottom of the tube was recovered. In a separate tube, the aqueous phase was treated once again with $2 \%$ Triton X-114 and, after centrifugation, the detergent phase was discarded. After separation, the Triton X-114 and buffer were added, respectively, to the aqueous and detergent phases to obtain equal volume, and the same salt and detergent content in both samples. The procedure described was applied to samples of the Triton X-100 insoluble membranes, the mucosal membranes preparation (11), and the affinitypurified MBP described previously $(11,12)$.

\section{Nitrous Acid (HONO) Deamination of MBP}

The $97 \mathrm{kDa} \mathrm{MBP}$, isolated by affinity column chromatography $(11,12)$ and the $97 \mathrm{kDa}$ protein extracted from apical membranes with Triton X-114 were subjected to deamination reaction $(23,24)$. The proteins, pre-extracted with chloroform/methanol (2:1, volume per volume; v/v), were dissolved in four volumes of $0.1 \mathrm{M}$ sodium acetate, $\mathrm{pH} 3.5$, and one volume of sodium nitrite or sodium nitrate (mock hydrolysis), adjusted with $6 \mathrm{~N} \mathrm{HCl}$ to $\mathrm{pH} 4.0$, and incubated at $50^{\circ} \mathrm{C}$ for $4 \mathrm{hr}$. The released lipids were extracted with three volumes of chloroform/ methanol/HCl (2:1:0.01, v/v/v), and twice with three volumes of chloroform saturated with water. The or- 
ganic solvent extracts were evaporated and subjected to thin layer chromatography in the solvent system consisting of chloroform/ methanol/water $(65: 35: 5, v / v / v)$. The water phase of the samples was subjected to Triton X-114 partition and SDSPAGE (22).

\section{Dissociation of Mucin and Treatment with Bacterial Hydrolases}

Dissected stomachs were rinsed with saline or with saline containing $1.0 \%$ Triton X-100. The surface of the stomachs was blotted with filter paper, cut into two mirror images, and transferred to small culture dishes containing the $H$. pylori growth medium, H. pylori spent medium, bacterial phospholipase C (PLC), or saline. The incubation was performed with and without protease inhibitors (20). After 60 min in the tissue incubator, the halves of the stomachs were used for preparation of apical membranes $(20,25)$, and MBP extraction with Triton X-114 (22).

\section{Quantitation of Binding Between Mucin and MBP}

The Triton X-114 extract prepared from the gastric mucosa apical membranes, isolated after the incubation described above, was diluted with buffer to $200 \mu \mathrm{l}$, and cooled in an ice bath. When dissolved, $150 \mu \mathrm{l}$ was applied to microplate wells, diluted out with carbonate buffer, incubated overnight at $4^{\circ} \mathrm{C}$, and blocked with buffer containing $0.2 \%$ serum albumin. Mucin collected from the respective stomachs before incubation was introduced to the blocked wells at concentration of one absorbance unit in the reaction with wheat germ agglutinin (WGA) lectin that on average corresponded to $0.125 \mathrm{mg}$ mucin $/ \mathrm{ml}$ $(26,27)$. Another set of samples consisted of the same mucin preincubated with the soluble MBP generated by the deamination reaction with HONO (23). The plates were incubated overnight at $4^{\circ} \mathrm{C}$, the wells rinsed with blocking buffer, and the mucin binding quantitated $(26,27)$. For the controls, the MBP and mucin were incubated with water-soluble proteins of gastric mucosal extract remaining in the supernatant after sedimentation of the Triton X-100insoluble apical membranes. After incubation, the binding of mucin to MBP was quantitated with WGA lectin enzyme linked lectin binding assay (ELLA) assay described above and in $(26,27)$. In parallel experiments, the aliquots of Triton X-114 extracts were subjected to SDS-PAGE, and staining with Coomassie and carbohydrate-specific stain (22).

\section{Preparation of Mucin and Mucin Oligosaccharides}

Standard gastric mucin prepared from the mucosal scrapings of 10 rat stomachs was isolated by the exclusion column chromatography on Bio-Gel A-50 (19) (Bio-Rad Laboratories, Inc., Richmond, CA). The same isolation technique was applied to mucin preparations from the individual rat stomachs. Thus prepared, samples were subjected to $\beta$-elimination reaction (28). The released oligosaccha-rides were quantitated using WGA in ELLA assay $(26,27)$. The oligosaccharides released from mucin by the $\beta$-elimination reaction were used in the binding studies with MBP without further separation, or subjected to thin layer chromatography in the solvent mixture consisting of n-butanol/acetic acid/water $(5: 3: 2, v / v / v)$, and separated into three major fractions consisting of oligosaccharides with migration corresponding to monohexasaccharide (fraction I), octa-decasaccharide (fraction II), and oligosaccharides remaining close or on the origin of the plate (fraction III).

\section{Identification of the Mucin Oligosaccharides Binding to $M B P$}

The MBP $(0.1-0.2 \mathrm{mg} / \mathrm{ml})$ and the oligosaccharides released from mucin (corresponding to $0.8 \mathrm{mg}$, mucin in case of the experiments containing the entire spectrum of oligosaccharides, or the entire fraction recovered after thin-layer chromatography of the material corresponding to $1.0-1.2 \mathrm{mg}$ of mucin) were combined with $0.5 \%$ Triton X-114 (22). The clear samples were incubated for $5 \mathrm{~min}$ at room temperature, overlaid on sucrose cushion, and incubated for $5 \mathrm{~min}$ at $30^{\circ} \mathrm{C}$. After the clouding occurred, the oily droplet of the detergent phase was recovered, and the material subjected to nitrous acid deamination reaction and second partition with Triton X-114. The material recovered in the water phase was applied to a thin-layer plate and subjected to chromatography in the solvent system consisting of n-butanol/acetic acid/water (5:3:2, v/v/v). After $6 \mathrm{hr}$ of ascending chromatography, the plate was dried, the area corresponding to application zone was scraped off, and the plate sprayed with orcinol reagent $(28)$.

\section{Results}

The MBP of gastric mucosal membranes prepared by affinity chromatography on WGA-Sepharose column (Sigma Chemical Co St. Louis, MO) $(11,12)$, and the proteins isolated from the Triton X-100-insoluble apical membranes of gastric mucosa that were partitioned into Triton X-114 detergent phase and on SDS-PAGE migrated as a $97 \mathrm{kDa}$ glycoprotein (Fig. 1). The partition of the mucosal membrane protein into Triton X-114 suggested that the isolated integral glycoprotein was anchored in the mucosal membrane by glycosylphosphatidylinositol (GPI) moiety (29). The results of MBP hydrolysis with nitrous acid provided subsequent evidence that confirmed our contention. Upon hydrolysis, the MBP partitioned into the aqueous phase of Triton X-114 (Fig. 2B), whereas, the organic solvent extract con-tained lipids that migrated as acylated derivatives of phosphatidylinositol on thin layer chromatography (Fig. 3).

The protein released from MBP with HONO stained with carbohydrate detecting reagent and its 


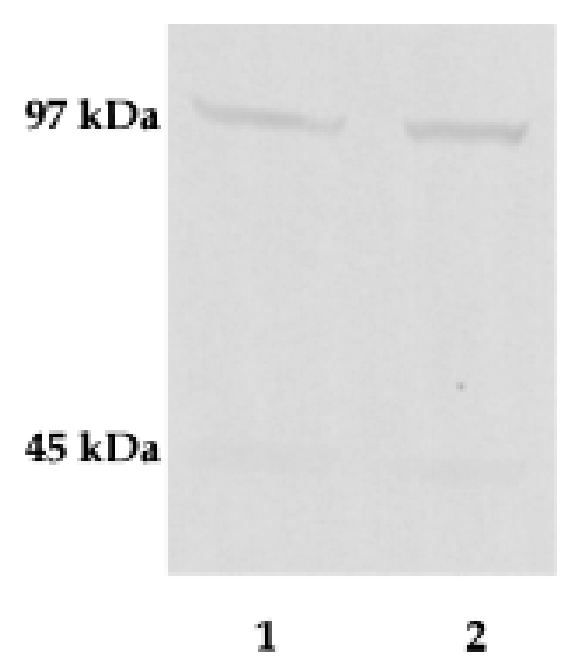

Fig. 1. Mucin binding protein (MBP) of rat gastric mucosa. Lane 1, the MBP from Triton X-114 extract

of the apical gastric epithelial membranes insoluble in $1 \%$ Triton X-100 and separated on sucrose gradient as described in "Materials and Methods." Lane 2, the Triton X-114 extract of the MBP purified from rat gastric mucosa by affinity column chromatography as described in (11). The samples were detected using Immun-Blot kit for glycoprotein detection from Bio-Rad.
A

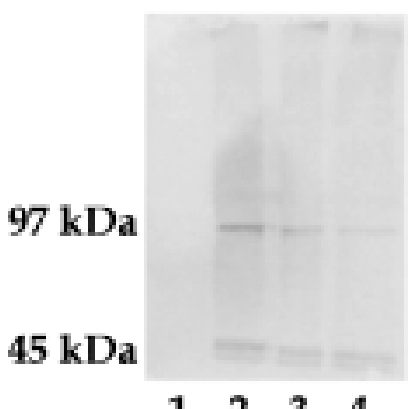

B

\section{$97 \mathrm{KDa}$}

\section{$45 \mathrm{KDa}$}

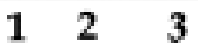

Fig. 2. Hydrolysis of mucin binding protein (MBP). (A) Nitrous acid hydrolysis and partition of rat mucosal MBP in Triton X-114 (22,23). Lane 1, Triton X-114 extract of MBP treated with nitrous acid (HONO). Lane 2, Triton X-114 extract of MBP treated with $\mathrm{HONO}_{2}$ (mock hydrolysis). Lanes 3 and 4, the aliquots of MBP before incubation in $\mathrm{HONO}$, and $\mathrm{HONO}_{2}$, respectively. (B) Water phase from the MBP preparations treated with HONO, corresponding to lanes $1-3$ in panel A. The protein transferred from SDS-PAGE to cellulose membrane was stained using Immun-Blot kit for glycoprotein detection (BioRad). The carbohydrate labeling with biotin was performed with protein immobilized in nitrocellulose.

migration on SDS-PAGE remained unchanged. The analysis of the lipids released with HONO showed the presence of inositol-containing phospholipids that migrated on high performance thin layer chromatography (HPTLC) plate faster than the nonacylated phosphatidylinositol (PI) standard (Fig. 3). These

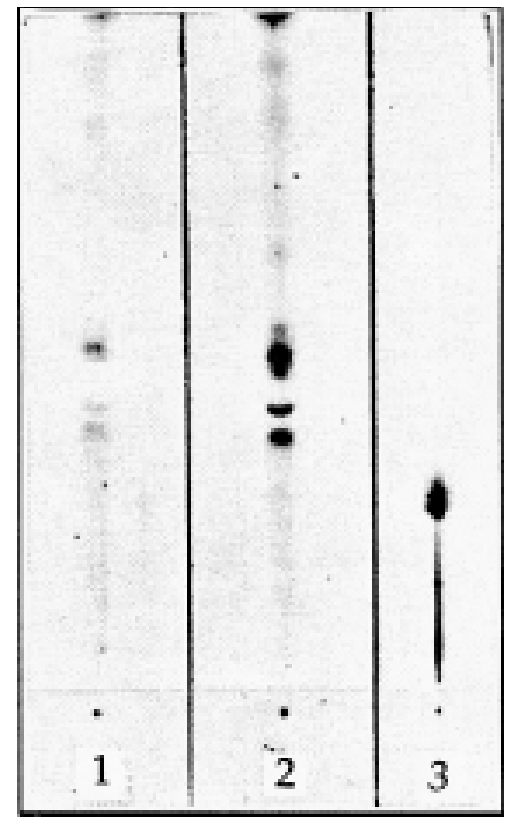

Fig. 3. The thin-layer chromatography of the lipids extracted from the samples of mucin binding protein (MBP) subjected to nitrous acid (HONO) hydrolysis. Lane 1, lipids released from the MBP isolated by wheat germ agglutinin (WGA)-affinity column (11). Lane 2, lipids released from the MBP obtained from Triton X-100-insoluble mucosal membranes. Lane 3, phosphatidylinositol standard. The same samples subjected to mock hydrolysis with $\mathrm{HONO}_{2}$ afforded organic solvent extract free of lipids. The thin-layer chromatography of the chloroform/methanol/HCl $(2: 1: 0.01, \mathrm{v} / \mathrm{v} / \mathrm{v})$ extract of the hydrolysis products with $\mathrm{HONO}$ and $\mathrm{HONO}_{2}$ was performed on high performance thin layer chromatography (HPTLC) silica gel 60 plates in chloroform/methanol/water $(65: 25: 5, \mathrm{v} / \mathrm{v} / \mathrm{v})$ and visualized by charring with $30 \%$ ammonium bisulfate.

lipids were not released when mock hydrolysis with $\mathrm{NaNO}_{2}$ was performed. The fact that the inositol-containing phospholipids were released only by nitrous acid hydrolysis, provided evidence that MBP of gastric mucosal apical surfaces was the GPI-anchored integral membrane component $(21,23,29)$.

The preparations of MBP from the membranes without prior Triton X-100 treatment afforded MBP associated with mucin (Figs. 4A and 5); whereas, pre-extraction of the membranes with $1 \%$ Triton X-100 afforded preparations depleted of mucin (Fig. $4 \mathrm{~B}$, lanes 5-7). The mucin-free MBP preparations were susceptible to hydrolysis with pepsin and H. pylori protease, but not to PLC digestion. In situ, neither pepsin nor the $H$. pylori protease cleaved the exoprotein of MBP (Fig. 6A, lane 1). However, if the gastric mucosal surface was subjected to Triton X-100 extraction, the MBP was degraded with pepsin as well as with $H$. pylori-derived protease (Fig. 6A, lanes 4-7). With the degradation of mucosal MBP, the binding of mucin to the gastric epithelial surface also ceased (Fig. 6A, lanes 5 and 7). 
A



B

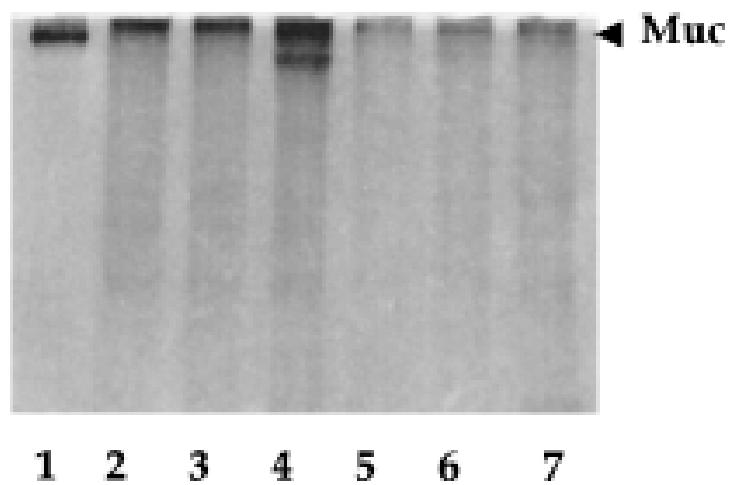

Fig. 4. Mucin mucin binding protein (MBP) complexes from rat gastric mucosa. Gastric mucosal membranes prepared as described in (11) or by $1 \%$ Triton X-100 treatment (20) were subjected to Triton X-114 partition, SDS-PAGE, and immunoblotting. (A) Silver-stained SDS-PAGE gel of six separate preparations of MBP as in (11). Lane 7, purified rat gastric mucin (Muc). (B) Silver-stained SDS-PAGE gel of the samples obtained from the membranes isolated as in (11) or after $1 \%$ Triton extraction (20) and the transfer of MBP to nitrocellulose. Lane 1 depicts purified rat gastric mucin. Lanes 2-4 show the samples, as in the lanes $1-3$, panel A. Lanes 5-7 depict material obtained from the mucosal membranes treated with $1 \%$ Triton X-100.

Thus, the experiments suggest that without mucin bound to MBP the exposed portion of MBP peptide is subjected to digestion by mucosal own proteolytic enzyme (pepsin) and H. pylori protease. In contrast, the unprotected MBP of rat gastric mucosa was not susceptible to degradation with bacterial PLC (Fig. 6B). After digestion with PLC, the material remained extractable with the Triton X-114 and MBP was not released into the water phase.

The binding experiments conducted with soluble MBP and mucin in ELLA system showed that preincubation of mucin with MBP prevented its at-

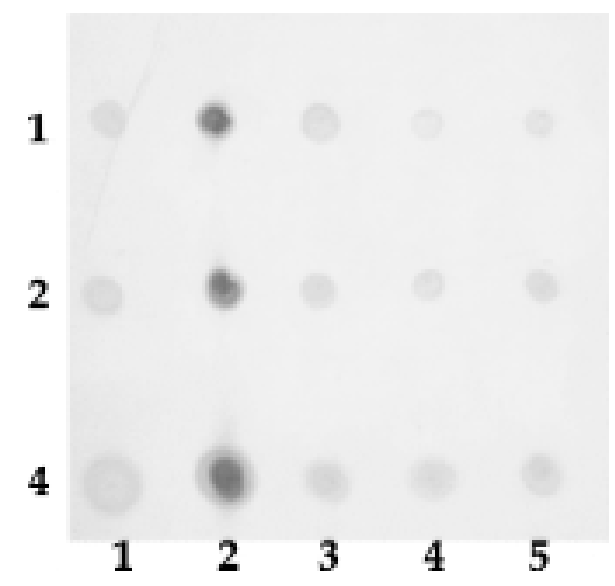

Fig. 5. Mucin identification by dot-blot staining with 3 G12 mucin monoclonal antibody $(15,19)$.

The material extracted from gastric mucosal membranes (11) before SDS-PAGE (lane 1), the purified rat gastric mucin (lane 2 ), and the mucin extracted from the SDS-PAGE samples shown in Figure 4A, lanes 4-6 was applied in Figure 5, lanes 3-5, respectively. The numbers on the left represent amount of material applied; $5 \mu \mathrm{l}$ (1), $10 \mu \mathrm{l}$ (2), and $20 \mu \mathrm{l}$ (4) per dot. tachment to the solid phase MBP (Fig. 7A, closed triangles). On the other hand, the preincubation of the well-bound MBP with the soluble proteins of the mucosal epithelial cells did not inhibit formation of the complex between mucin and the solid phase MBP (Fig. 7A, solid squares). These results and the data obtained with six independent preparations depicted in Figure 7B, allowed us to conclude that the affinity between mucin and MBP was specific, reproducible, and not replaceable by other cellular proteins.

The involvement of mucin oligosaccharides in the binding with MBP was first suggested when, after partial deglycosylation, mucin lost its affinity for MBP (11). This was reaffirmed by the incubation of MBP with the oligosaccharides of mucin released by the $\beta$-elimination reaction (28). The results of incubation of MBP with O-glycosidicallinked oligosaccharide of mucin revealed that octaand decasaccharides, and possibly larger oligosaccharides, were MBP ligands (Fig. 8). Carbohydrate units smaller than octasaccharide were not detected in the complex. We have not yet determined which terminal carbohydrate sequence and anomeric configuration was responsible for the binding. But, from the results on binding of mucin derived from alcohol-fed rats, it could be surmised that the nonreducing terminal configuration was the essential element for the binding with MBP, as the less glycosylated mucin produced by ethanol-fed rats displayed up to $70 \%$ reduction in binding to its MBP (13).

The evidence that alcohol interfered with posttranslational glycosylation in Golgi and the data revealing substantial reduction in binding of ethanolderived mucin to MBP provided a connection between the role of the specific carbohydrate determinants and mucin adherence to the mucosal sur- 
A

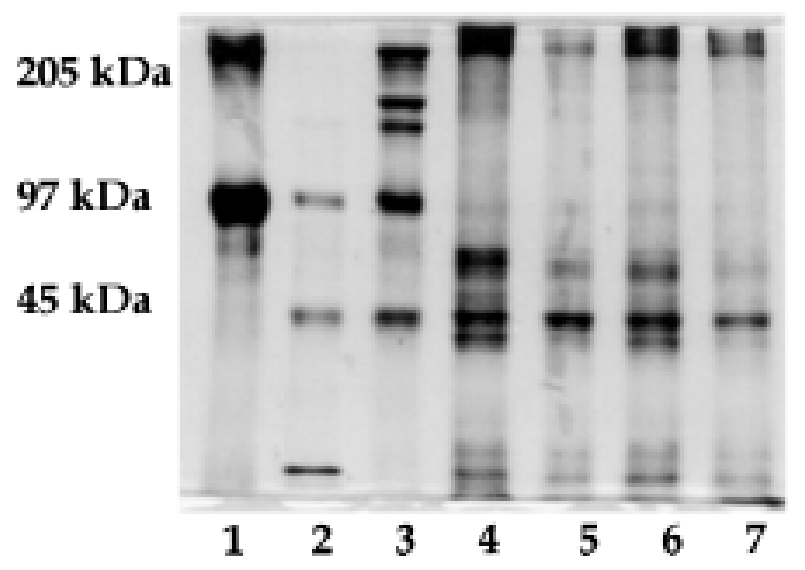

B

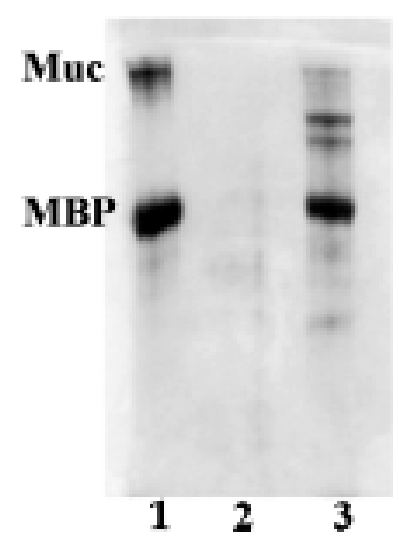

Fig. 6. Effect of the pepsin, Helicobacter pylori protease, and phospholipase C (PLC) on mucin binding protein (MBP) of rat gastric mucosa. The individual rat stomachs were rinsed with buffered saline, bisected to form identical halves of the organ, and subjected to extraction with saline or $1 \%$ Triton X-100, incubation with or without protease inhibitors, and incubation with the mediumderived from H. pylori culture (11). (A) Lane 1, MBP-mucin complex extracted from the gastric mucosa, not extracted with Triton X-100, and incubated in the presence of protease inhibitors. Lanes 2 and 3, molecular weight markers. Lanes 4-7, the preparations of the MBP from the stomach halves treated with $1 \%$ Triton X-100 and incubated without protease inhibitors in saline (lanes 4,6$)$ and with medium from H. pylori culture (lanes 5,7). (B) Effect of PLC on the partition of MBP to Triton X-114. Lanes 1 and 3, Triton X-114 phase from the MBP before and after PLC treatment, respectively. Lane 2, water phase from the partition of PLC-treated MBP. The gel in panel A was stained with Coomassie stain. Panel B represents the Western blot containing mucin (Muc) and main binding protein stained with ImmunBlot kit for glycoprotein detection from Bio-Rad (Richmond, CA).

face. Whether the interaction of mucin with MBP that diminished with alcohol feeding totally was responsible for development of the unprotected gastric epithelium was not known. However, the insufficiency in covering the epithelial surface $(13,15,16)$ may create the opportunity for binding of the agents that, with mucin in place, could not approach the epithelial surface, degrade the exoproteins of the apical membrane, and generate further changes that may perturb the normal function of the epithelium.

\section{Discussion}

The evidence presented in this work provides further insight to the concept of mucosal protection. It augments the view of the mucosal barrier as a layer of viscous mucin that, by the physical presence, prevents microbes and toxins from direct contact with the cell $(30,31)$ to the entity that is in a specific interaction with an integral protein of the apical epithelial membrane. In this study, we focused on investigation of the elements that are essential for the interaction and adherence of mucin to the apical mucosal surfaces. The initial analysis of the epithelial membrane proteins shows that the $97 \mathrm{kDa}$ protein from gastric mucosa displays carbohydratespecific affinity with mucin (11). In the work presented in this paper, we elaborate further on the concept, provide further characterization of MBP, and determine the affinity ligand on mucin responsible for the interaction.

We demonstrate that the MBP is concentrated in the apical gastric epithelial membranes and repre- sents an integral membrane protein $(21,29)$. Its extraction with Triton X-114 provides tentative evidence that it belongs to the group of membrane proteins containing a GPI anchor. This feature of MBP is firmly established by the release of phosphatidylinositols (PIs) in the deamination reaction with nitrous acid (23). Following nitrous acid deamination, the released lipid anchor is extracted with an acidified chloroform-methanol mixture and MBP partitions to the aqueous phase of the detergentcontaining biphasic system $(23,24)$. Hence, we conclude that Triton $\mathrm{X}-114$ partitioning protein from the mucosal apical epithelial membranes, which is insoluble in Triton X-100 and previously was isolated by the affinity chromatography (11), is a GPI-anchored integral membrane protein that resides in the apical surface of gastric mucosa.

The interaction of MBP with mucin that appears to depend on carbohydrate determinants located on the $\mathrm{O}$-glycosidical-linked oligosaccharides of mucin $(11,13)$ is further characterized. The significance of the specific mucin carbohydrate chains in mucin binding to MBP is ascertained by the analyses employing mucin-derived oligosaccharides. Three major fractions of the oligosaccharides were introduced to the reaction mixture with MBP, partitioned with Triton X-114, and the detergent phase subjected to thin-layer chromatography. The analysis reveals that octa- to decasaccharides are retained with MBP. The affinity for MBP also is observed with larger gastric mucin oligosaccharides that remain in the fraction consisting of the saccharides remaining near and at the origin of the thin-layer plate, but the shorter 
than octasaccharide chains are not binding. These results suggest that the assembly of the link between MBP and gastric mucin depends on posttranslational terminal glycosylation of mucin occurring on the saccharides consisting of at least six to seven carbohydrate residues.

Indeed, as we demonstrate in the studies on individual animals, the changes in mucin induced by chronic ethanol diet cause a dramatic decline in the interaction of mucin with MBP $(13,16)$, and mucin retention on gastric mucosal surfaces $(14,15)$. Together with the earlier studies that revealed ethanolinduced changes in posttranslational glycosylation and elongation of mucin oligosaccharides (15), it appears that MBP binding with mucin is affected by the changes in carbohydrate metabolism, particularly by changes in the oligosaccharides elongation (13-16). Furthermore, the experiments with mucin and MBP from the individual animals provides evidence that ethanol-induced changes in MBPmucin binding are invariant, although the impact of ethanol on the interaction of mucin with MBP is unequal in time $(13,16)$.
The role of MBP in mucin binding was studied also by employing quantitative ELLA assay $(26,27)$. As shown in Fig. 7, the solid phase-bound MBP and its interaction with soluble mucin show constancy and reproducibility. The preincubation of gastric mucin with the soluble, nitrous acid-released exoprotein of MBP abolished mucin binding to the solid phase MBP. In contrast, preincubation of mucin or MBP with the soluble cytosolic proteins of the mucosal cells does not interfere with the ability of mucin and MBP to interact. Thus, we conclude that the retention of mucin on the apical epithelial surface is specific and controlled by the structure of mucin oligosaccharides and MBP.

Until now, beyond the acknowledgment of the antigenic blood group determinants, the relevance of carbohydrate chain completion on gastric mucin was not contemplated as an important functionrelated characteristic. Even though, the earlier studies on mucin sulfation showed that the agents affecting processing of carbohydrate substrates alter posttranlational modification and function of mucin (32) and other cell surface glycoconjugates (33), the
A

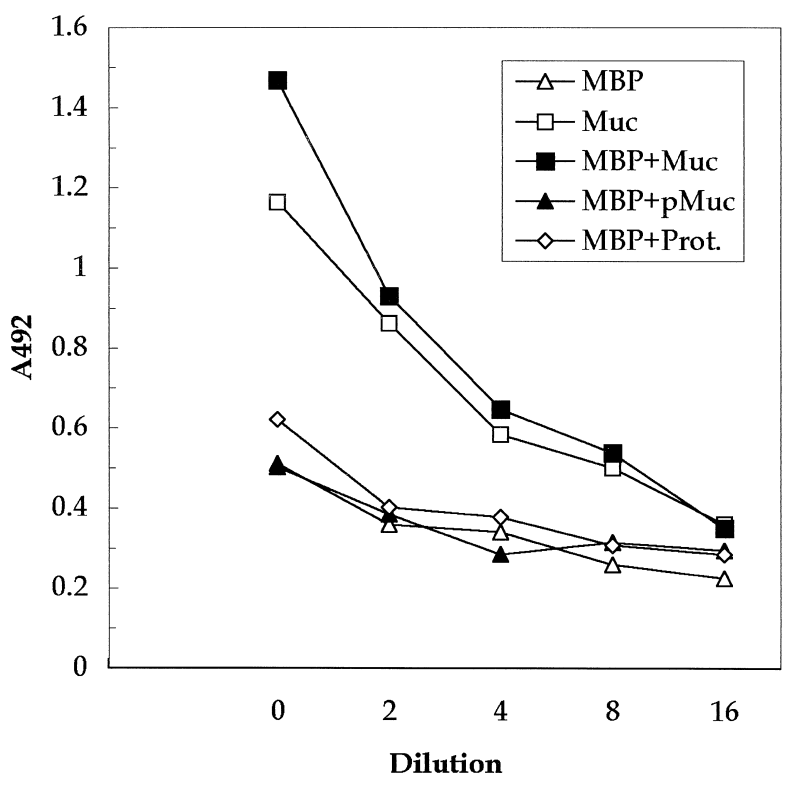

B

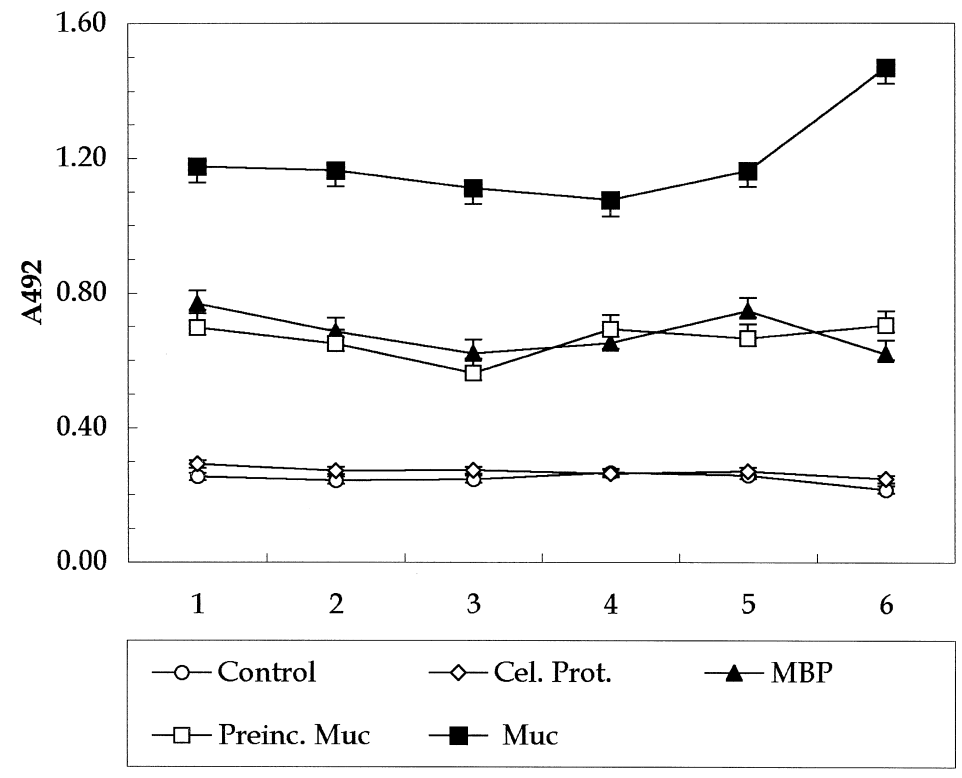

Fig. 7. Binding of mucin (Muc) and mucin binding protein (MBP). (A) Quantitation of mucin binding protein (MBP) and mucin and their binding in the Enzyme linked lectin binding assay (ELLA) system according to method of Rhodes and Ching (26,27). The amount of each sample was determined by the wheat germ agglutinin (WGA)-binding assay. The binding of MBP and mucin to solid phase was determined at the range of dilutions shown in Figure 7A. On the average, of the total $1 \mathrm{~A}_{492} / 100 \mu \mathrm{l}$ of purified MBP and mucin, $45 \%$ and $80 \%$ bound to the solid phase, respectively. The binding of MBP is depicted by open triangles and mucin (Muc) by open squares. The binding of mucin to solid phase MBP pretreated with the soluble mucosal protein (MBP + Muc) is depicted by solid squares; the binding of mucin preincubated with soluble MBP at concentration of $1.0 \mathrm{~A}$ at $492 \mathrm{~nm}$, and then to solid phase-bound MBP $(\mathrm{MBP}+\mathrm{pMuc})$ is shown by solid triangles; the binding of the mucosal soluble protein to MBP (MBP + Prot.) is shown by the open diamonds. The concentration of purified mucin used was $0.125 \mu \mathrm{g} / 100 \mu \mathrm{l}$ and the soluble proteins from gastric mucosal preparation was $100 \mu \mathrm{g} / 100 \mu \mathrm{l}$. (B) Binding of mucin and MBP carried out with six preparations from gastric mucosa of individual rats. The binding of mucin to solid phase MBP (Muc) is shown by solid squares and the mucin preincubated with soluble MBP (Preinc. mucin) is shown by open squares. The amount of MBP attached to solid phase is shown by solid triangles (MBP), the overall binding of the soluble cellular proteins (Cel. Prot.) to the plate is shown by open diamonds, and the background readings (Control) are shown by open circles. The results presented depict six independent experiments. The error bars depict the average $(6 \%)$ variations in binding performed in triplicate on the same titration plate. 


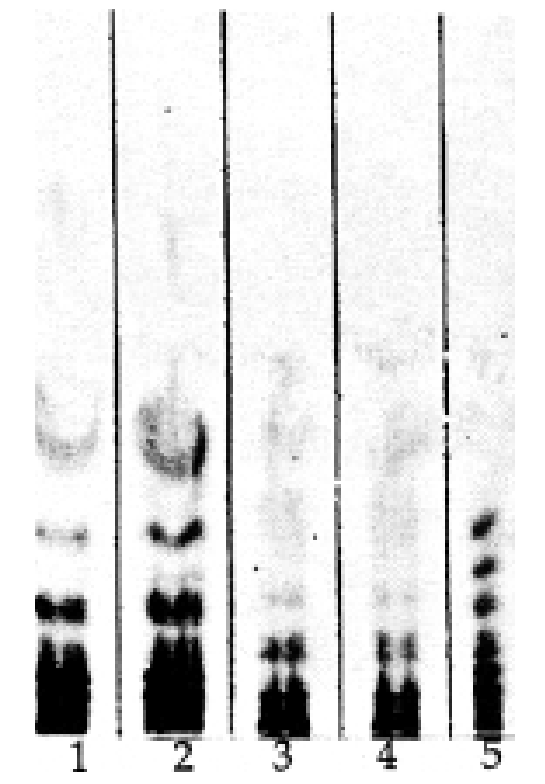

Fig. 8. Thin-layer chromatographic separation of the oligosaccharides released from rat gastric mucin by $\beta$-elimination reaction. Those remaining with mucin binding protein (MBP) following partition into Triton X-114 are as described in the "Materials and Methods." The material from incubation of MBP with oligosaccharides that partitioned into Triton X-114 was hydrolyzed with nitrous acid (HONO) and the sample for thin layer chromatography was recovered from aqueous phase of the second partition with the detergent. The water soluble phase was applied to thin layer plate and developed in solvent system consisting of n-butanol/ acetic acid/water (5:3:2, v/v/v). After 6 hr of ascending chromatography, the plate was dried and sprayed with orcinol (28). Lanes 1 and 2 represent oligosaccharides recovered in the water phase of the first partition of MBP incubates with Triton X-114. Lanes 3 and 4 contain the water phase of the HONO hydrolyzed Triton X-114 extract of MBP. Lane 5 represents the oligosaccharides mixture released from rat gastric mucin by $\beta$-elimination reaction (28). The HONO hydrolysis of the Triton X-114 extract of MBP incubated with mucin oligosaccharides was instituted to remove the sample from the Triton X-114 that interfered in the thin layer chromatography. The analyses reveal that the oligosaccharides interacting with MBP are mainly in the fraction contained in region of octa- to decasaccharides or larger oligomers. As apparent from the lanes 1 and 2, the smaller oligosaccharides are not retained with MBP samples and are not binding, even if introduced as separate fraction (unpublished results).

consequences of the changes in gastric mucosa were not deemed significant. By the same token, the function of Golgi transporters and the process of substrate delivery for posttranslational glycosylation in the final processing of the mucin substrate (34-36) was not considered relevant. Our results, however, show that ethanol induced decrease in glycosylation affects the formation of MBP-mucin complex that is necessary to cover and separate the gastric epithelial cells from agents in the gastric contents. Whether the reduced interaction of mucin with the epithelial protein is a sole determinant of the increased opportunity for microbes and toxins to act upon gastric epithelium is not yet known. However, the initial findings on proteolytic susceptibility of MBP exoprotein and concomitant loss of the ability to interact with mucin strongly support the notion that the complex is highly relevant in preservation of gastric mucosal barrier.

In situ, the degradation of MBP is not detectable when mucin remains on the gastric surface or when the detergent-treated mucosa is preincubated with standard mucin prior to the digestion with pepsin or $H$. pylori protease (unpublished results). Even without proteolytic degradation of MBP, the release of mucin from gastric epithelial surface, whether induced by chemical agents disrupting the complex or caused by inadequate glycosylation, may expose the gastric epithelial MBP to the action of agents, such as lipopolysaccharide (LPS) (12). Therefore, it remains viable that displacement of mucin from the cellular surface, even without release of MBP exodomain, creates the opportunity for the LPS with mucin-mimicking oligosaccharides to bind to MBP (12) and signal LPS-mediated intracellular processes (37). The evidence obtained by others $(17,18)$ on mucin-carbohydrate mimicking structures in bacterial LPS and our results that, in the absence of mucin, LPS binds to MBP (12) strongly suggest that the presence of mucin in complex with MBP is extremely important in the continuously challenged environment of microbes. Without mucin interacting with the MBP, the bacterial LPS has the opportunity to bind to epithelial cell and stimulate LPS-specific responses (37). Further studies are needed to determine the LPSmediated signaling and its interference with the protective mucin-mediated processes. With LPS in complex with MBP instead of mucin, the epithelial surface is not only receiving untoward LPSgenerated signals, but is uncovered and exposed to the ravages of microbes, toxins, and uncountable agents present in gastric contents (38).

The presence of GPI moiety in MBP determines its anchoring in the epithelial membrane. In rat gastric mucosa, the GPI anchor appears to contain an acylated inositol that on HPTLC is reflected in a faster-than-the-PI-standard mobility and according to others (24) renders GPI-anchored proteins resistant to bacterial PLC. Based on the evidence presented in Roberts et al. (24), the thin-layer chromatographic migration of the nitrous acid-released, PIs of gastric MBP and the resistance of the MBP anchor to the bacterial PLC, we suggest that rat gastric mucosal MBP contains PI with the inositol substituted with fatty acids. Such a structural asset of MBP provided resistance to bacterial PLC and, possibly, an explanation as to the imperviousness of rats and nonhuman primates mucosa to $H$. pylori colonization (39). Whether this is indeed the exclusive structural asset providing rat gastric MBP with the resistance to PLC and, thus, with exceptional or complete protection from $H$. pylori infection is not known and requires further investigation. 
The evidence on the specificity of mucin-MBP binding supports the concept that mucin complexed to its MBP guards the mucosa from foreign influences and, thus, the opportunity for the stimulation of immune system arises only when the complex is not generated and mucin is not occupying its sites $(7-9,41)$. Therefore, most oral vaccines successful at stimulating mucosal immunity require irritating mucin displacing adjuvents (41). With mucin in the complex with MBP, neither the surface-oriented action of the lumenal microbes nor their transfer to circulation and the production of IgA is attained.

The fact that ethanol interferes with glycosylation of mucin and induces susceptibility to infection (13-15,42) provides further, although indirect, evidence that supports our concept on the role of mucin-MBP epithelial boundary that is the innate protective barrier of the epithelial surfaces. The studies on ethanol-induced impairment of the mucosal barrier assembly provoke speculation that the protection of epithelial surfaces could be manipulated and improved by providing the carbohydrate substrates necessary for growth of the oligosaccharides involved with mucin binding to MBP. Since the process of oligosaccharide elongation is affected by metabolic status of cells, the dietary insufficiencies or the illness-induced changes would be manageable, and the life-threatening infections reduced or eliminated. Certainly, the initial innate defense would decrease contact and infection of the mucosal surfaces, provocation of the undesired stimulation of cellular epithelial processes, and alleviate the burden for humoral responses of the immune system $(7-9,43,43,44)$.

The specific innate mechanism that determines microbial clearance before mucosal infection and tissue invasion ensues is as significant as the intact epidermis to the protective function of the skin. An optimization of the conditions to built innate immunity reflected in the specific mucosal protection may have a major impact on the prevention of the infections through oral, nasal, gastrointestinal, pulmonary, or urogenital surfaces. It seems a far better strategy to built the protective barrier, rather than resort to the repeated application of monoclonal antibodies to the mucosal surface, which act only on the single type of pathogen trapped in mucus $(41,43)$. Further studies are needed to determine the specificity of the mucin-MBP interaction, variance in humans, and the role of diet in the posttranslational modification of mucin reflected in the assembly of the carbohydrate epitopes necessary for the maintenance of the epithelial protective barrier, as well as its restoration.

\section{References}

1. Allen A. (1981) Structure and function of gastrointestinal mucus. In: Physiology of the Gastrointestinal Tract. Johnson LR (ed.) Raven Press, New York, pp. 617-639.
2. Forstner JF, Forstner GG. (1994) Gastrointestinal Mucus. In: Physiology of Gastrointestinal Tract. Johnson LR (ed.) Raven Press, New York, pp. 1255-1283.

3. Perez-Vilar J, Hill RL. (1999) The structure and assembly of secreted mucins. J. Biol. Chem. 274: 31751-31754.

4. Hawkey CJ, Hudson N. (1995) Mucosal injury caused by drugs, chemicals and stress. In: Gastroenterology (Bockus,) 5th edition. Haubrich WS, Schaffner F, Berk JE (eds.) Sounders Co., Philadelphia, pp. 656-699.

5. Slomiany BL, Slomiany, A. (1993) In: The Stomach, Physiology, Pathophysiology and Treatment. Domschke W, Konturek SJ (eds.) Springer-Verlag, Berlin, pp. 116-143.

6. Wolfe MM, Sachs G. (2000) Acid suppression: optimizing therapy for gastroduodenal ulcer healing, gastroesophageal reflux disease, and stress-related erosive syndrome. Gastroenterology 118(Suppl. 1): S9-S31.

7. Lamm ME. (1997) Interaction of antigens and antibodies at mucosal surfaces. Annu. Rev. Microbiol. 51: 311-340.

8. Ganz T. (1999) Defensins and host defense. Science 286: 420-421.

9. Tang Y-Q, Yuan J, Osapay G, et al. (1999) A cyclic antibacterial peptide produced in primate leukocytes by the ligation of two trunkated $\alpha$-defensins. Science 286: 498-503.

10. Crouch EC. (1999) Modulation of host-bacterial interactions by collectins. Am. J. Respir. Cell Mol. Biol. 21: 558-561.

11. Slomiany BL, Piotrowski J, Czajkowski A, Murty VLN, Slomiany A. (1993) Characterization of gastric mucosal mucin receptor. Biochem. Mol. Biol. Int. 31: 745-753.

12. Piotrowski J, Slomiany A, Slomiany BL. (1993) Inhibition of gastric mucosal mucin receptor by $H$. pylori lipopolysaccharide. Biochem. Mol. Biol. Int. 31: 1051-1059.

13. Slomiany A, Skrodzka D, Piotrowski E, Slomiany BL. (1997) Alcohol-induced decrease in mucin binding to gastric mucosal receptor. Alcohol. Clin. Exp. Res. 21: A294

14. Takagi A, Slomiany BL, Kosmala M, Slomiany A. (1986) The synthesis and secretion of gastric mucus glycoprotein by mucosal cells exposed to ethanol. Biochim. Biophys. Acta 884: 1-10.

15. Slomiany A, Morita M, Sano S, Piotrowski E, Skrodzka D, Slomiany BL. (1997) Effect of ethanol on gastric mucus glycoprotein synthesis, translocation, transport, glycosylation, and secretion. Alcohol. Clin. Exp. Res. 21: 417-423.

16. Slomiany A, Piotrowski E, Slomiany BL. (1999) Further characterization of mucin binding receptor from rat gastric mucosa. Alcohol. Clin. Exp. Res. 23: A2.

17. Mandrell RE, Apicella MA. (1993) Lipo-oligosaccharides (LOS) of mucosal pathogens: mucosal mimicry and host modification of LOS. Immunobiology 187: 382-402.

18. Appelmelk BJ, Simons-Smit I, Negrini R, et al. (1996) Potential role of molecular mimicry between Helicobacter pylori lipopolysaccharide and host Lewis blood group antigens in autoimmunity. Infect. Immunity 64: 2031-2040.

19. Slomiany A, Okazaki K, Slomiany BL. (1992) Synthesis and macromolecular organization of gastrointestinal mucin. $J$. Clin. Gastroenterol. 14: S71-S81.

20. Sargiacomo M, Sudol M, Tang Z, Lisanti MP. (1993) Signal transducing molecules and glycosylphosphatidylinositollinked protein form a caveolin-rich insoluble complex I MDCK cells. J. Cell Biol. 122: 789-807.

21. Brown D, Rose JK. (1992) Sorting of GPI-anchored proteins to glycolipid enriched membrane subdomains during the transport to apical cell surface. Cell 68: 533-544.

22. Bordier C. (1981) Phase separation of integral membrane proteins in Triton X-114 solution. J. Biol. Chem. 256: 1604-1607.

23. Luhrs CA, Slomiany BL. (1989) A human membrane-associated folate binding protein is anchored by a glycosylphosphatidylinositols. J. Biol. Chem. 264: 2 1446-2 1449.

24. Roberts WL, Myher JJ, Kuksis A, Low MG, Rosenberry TL. (1988) Lipid analysis of the glycosylinositol phospholipid anchor of human acetylcholinesterase. J. Biol. Chem. 263: 18766-18775.

25. Diep DB, Nelson KL, Raja SM, Pleshak EN, Buckley JT. (1998) Glycosylphosphatidylinositol anchors of membrane glycoproteins are binding determinants for channel-forming toxin aerolysin. J. Biol. Chem. 273: 2355-2360. 
26. Rhodes JM, Ching CK. (1991) Serum diagnostic tests for pancreatic cancer. In: Cancer of the Pancreas. Clinical Gastroenterology Vol. 4. Neptolemos J (ed.) Bailliere Tindall, London pp. 833-852.

27. Rhodes JM, Ching CK.(1993) The use of lectins in the characterization of mucosal glycoproteins. In: Methods in Molecular Biology. Hounsell E (ed.) Humana Press, Totowa, NJ, pp. 247262.

28. Slomiany A, Zdebska E, Slomiany BL. (1984) Structures of the neutral oligosaccharides isolated from A-active human gastric mucin. J. Biol. Chem. 259: 14743-14749.

29. Brown DA, London E. (1998) Functions of lipid rafts in biological membranes. Annu. Rev. Cell Dev. Biol. 14: 11-136.

30. Slomiany BL, Slomiany A. (1991) Role of mucus in gastric mucosal protection. J. Physiol. Pharmacol. 42: 149-163.

31. Slomiany BL, Slomiany A. (1993) Mucus and mucosal protection. In: The Stomach. Domschke W, Konturek J (eds.) Springer-Verlag, Berlin, pp. 116-143.

32. Liau YH, Slomiany A, Slomiany BL. (1992) Role of sulfation in posttranslational processing of gastric mucin. Int. J. Biochem. 24: 1023-1028.

33. Safaiyan F, Kolset SO, Prydz K, Gottfridsson E, Lindahl U, Salmivirta M. (1999) Selective effects of sodium chlorate treatment on the sulfation of heparan sulfate. J. Biol. Chem. 274: 36267-36273.

34. Pugllielli L, Mandon EC, Rancour DM, Menon AK, Hirschberg CB. (1999) Identification and purification of the rat liver Golgi membrane UDP-N-acetylgalactosamine transporter. J. Biol. Chem. 274: 4474-4479.

35. Puglielli L, Hirschberg CB. (1999) Reconstitution, identifica- tion, and purification of the rat liver Golgi membrane GDPfucose transporter. J. Biol. Chem. 274: 35596-35600.

36. Hirschberg CB, Robbins PW, Abeijon C. (1998) Transporters of nucleotide sugars, ATP, and nucleotide sulfate in the endoplasmic reticulum and Golgi apparatus. Annu. Rev. Biochem. 67: 49-69.

37. Yang R-B, Mark MR, Gray A, et al. (1999) Toll-like receptor2 medilates lipopolysaccharide-induced cellular signalling. Nature 395: 284-288.

38. Marshall BJ. (1986) Campylobacter pyloridis and gastritis. J. Infect. Dis. 153: 650-657.

39. Dubois A, Berg DE, Incecik ET, et al. (1999) Host specificity of Helicobacter pylori strains and host responses in experimentally challenged nonhuman primates. Gastroenterology 116: 90-96.

40. Higgins LM, Frankel G, Connerton I, Goncalves NS, Dougan G, MacDonald TT. (1999) Role of bacterial intimin in colonic hyperplasia and inflammation. Science 285: 588-591.

41. Casadevall A, Scharff MD. (1995) Return to the past: the case for antibody-based therapies in infectious diseases. Clin. Infect. Dis. 21: 150-161.

42. Jerrells TR, Sibley D. (1995) Effect of ethanol on cellular immunity to facultative and intracellular bacteria. Alcohol. Clin. Exp. Res. 19: 11-16.

43. Zeitlin L, Cone RA, Whaley KJ. (1999) Using monoclonal antibodies to prevent mucosal transmission of epidemic infectious diseases. Emerging Infect. Dis. 5: 54-64.

44. Bisaillon M, Senechal S, Bernier L, Lemay G. (1999) A glycosyl hydrolase activity of mammalian reovirus $\mathrm{Ol}$ protein can contribute to viral infection through a mucus layer. J. Mol. Biol. 286: $759-773$. 